\title{
Braiding Simulation and Slip Evaluation for Arbitrary Mandrels
}

\author{
Remko Akkerman and Blasimir Hadir Villa Rodríguez \\ University of Twente, PO Box 217, NL-7500AE Enschede, The Netherlands \\ e-mail: r.akkerman@utwente.nl
}

\begin{abstract}
Braiding is a manufacturing process that is increasingly being used to manufacture pre-forms for Resin Transfer Moulding. A fast simulation method is presented for the prediction of the fibre distribution on complex braided parts and complex kinetic situations (e.g. changes in velocity, orientation). The implementation is suited for triangular surface representations as generated by many CAD software packages in use. Experimental results show that the results are sensitive to the friction conditions in particular regions. The friction conditions between the yarns and the mandrel are analysed, leading to the development of a slip indicator.
\end{abstract}

Keywords: textile composite fibre orientation friction.

PACS: 46.55.+d, 81.05.Qk

\section{INTRODUCTION}

Automated braiding is a suitable process for manufacturing reproducible preforms for resin transfer moulding (RTM). It provides a fast fibre lay-down due to the simultaneous fibre deposition. The highly interlaced structure of braids makes it possible to cover components with sharp curvatures and non-circular cross-sections, varying along the length of the component. Furthermore, the interlaced nature of braids provides high levels of impact strength. Typical examples of these RTM components are propeller blades, trailing arms for a helicopter landing gear (see fig.1) and automotive space frame components.

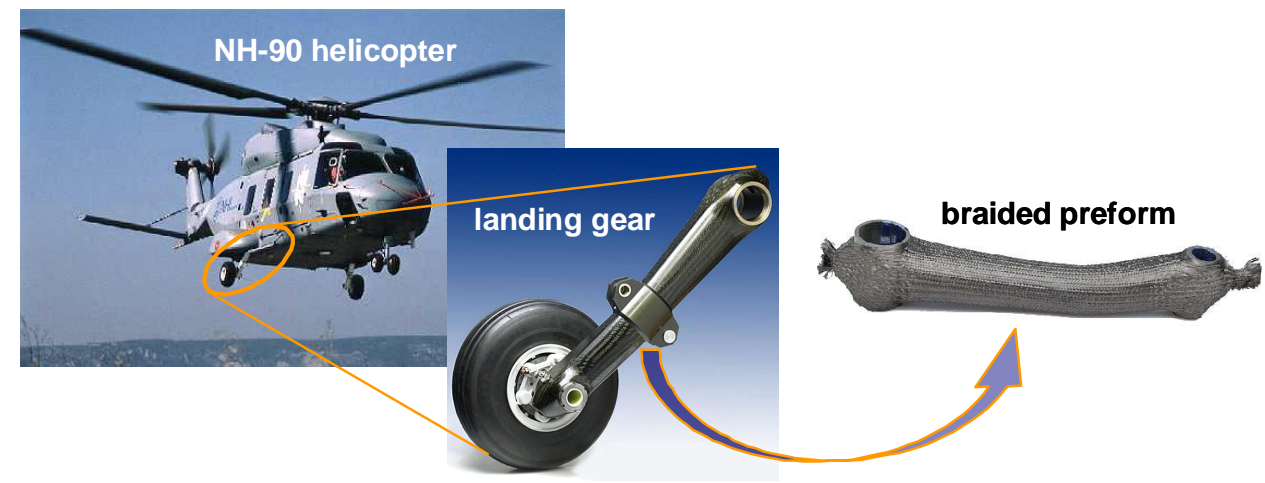

FIGURE 1. Braided preform for a composite landing gear (Courtesy Stork SP Aerospace). 
It is not trivial to predict the mechanical properties of an arbitrary braid reinforced product in advance, firstly because the lack of knowledge about the fibre orientation. Earlier research concentrated on continuous braiding on cylindrical cross sections $[1,2,3]$. Kessels and Akkerman [4] presented a model for the prediction of the yarn trajectories on 'complex shaped mandrels', with a non-axisymmetric cross-section, varying along the length of the component. This model was based on geometrical primitives (such as planar, cylindrical and spherical surfaces). A triangular surface mesh is a more versatile description. It is suitable for coupling process simulations to most CAD systems, as recently presented in [5]. Fibre slip is not included in this type of modelling, but was identified as an important factor to take into account. This paper summarizes the algorithms, results, experimental validation and the development of a slip indicator.

\section{PROCESS DESCRIPTION}

An illustration of a horn-gear braiding machine is given in fig.2. The mandrel, supported by a holder (not shown in the illustration), is located between the spools. The mandrel moves with an axial velocity, $V$. The yarns are driven by spools in the spool plane. One group of yarns, denoted as the warp yarns, moves clockwise while the weft yarns move counter-clockwise, both with an angular velocity of $\pm \omega$. The two yarn groups interlock, forming a closed biaxial fabric on the mandrel. Optionally a third group of yarns can be inserted through the horn gears (see fig.3). These stem yarns will be deposited in parallel to the mandrel axis, providing extra stiffness and strength in the axial direction of the now triaxially braided preform.

A pair of guide rings leads the yarns towards the mandrel. The yarns converge to the mandrel and touch the mandrel at a distance $H$ from the guide ring. The point where a yarn touches the mandrel is denoted as the fell point. In operation, the mandrel with its holder can be driven to the right and left alternately for both forward and reverse braiding, and the layer of braid formed previously is covered (or 'overbraided') by the newly formed one. In this manner, multilayered products can be braided in one run.

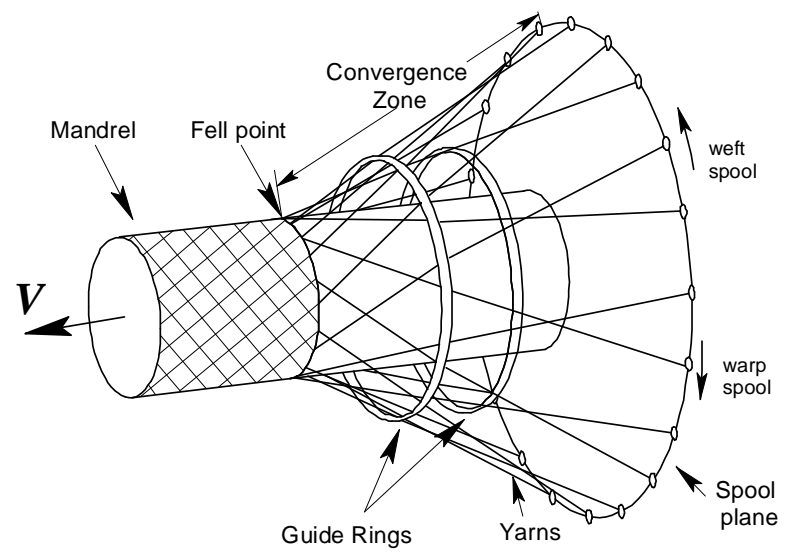

FIGURE 2. Braiding machine (schematically).

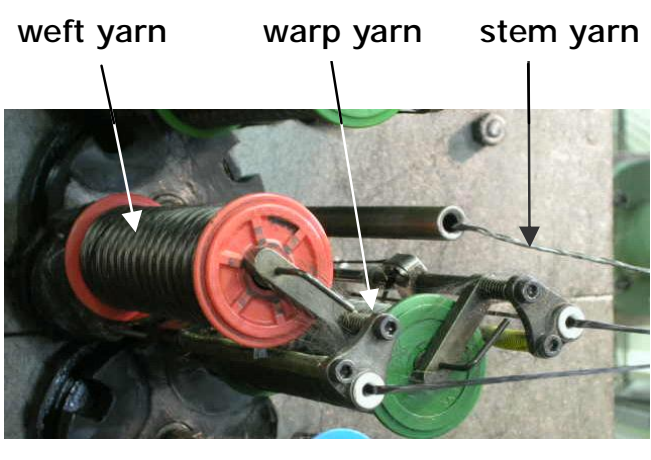

FIGURE 3. Yarn supply in the spool plane. 


\section{MATHEMATICAL MODEL}

Fig. 4 shows a model of a braiding machine with a complex mandrel. The frame of reference is chosen to be fixed to the mandrel. The yarn supply point, $\stackrel{q}{q}$, is located on the guide ring in this case. The figure further shows the fell point of the yarn, $p$, on the surface of the mandrel, $Q$, with for every point $\dot{x}$ on the surface:

$$
Q(\dot{x})=0 \text {. }
$$

The angle between the path of the yarn and the tangent line of the surface in $z$ direction js the braid angle, $\alpha$. The path of the yarn can be defined as a trajectory $f(\lambda)$ where the scalar $\lambda$ increases monotonically along the fibre path. The fell point, $p$, moves along this trajectory in time.

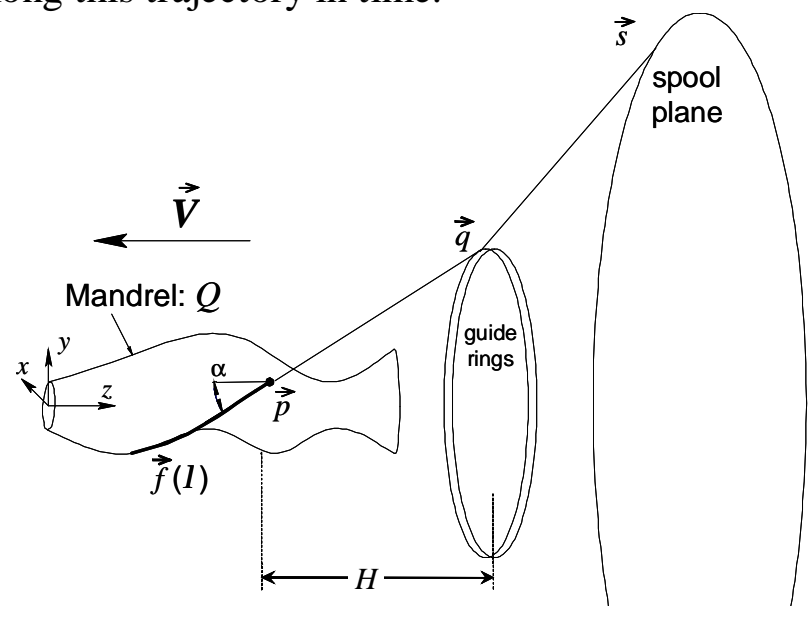

FIGURE 4. Model of a braiding machine with a complex mandrel.

The braiding process is simplified to enable fast modelling for use in subsequent numerical optimisation procedures. Zero friction is assumed before deposition of the fibres on the mandrel, while total interlocking (and hence zero slip) is assumed after deposition. This results in an approach in which each yarn can be described separately. The progression of the fell points of all yarns is predicted in discrete time steps. The yarn is continuous and differentiable at the fell point, where the free segment is connected to the deposited segment. This implies that the direction of the fibre path at the fell point, $p$, has to be equal to the direction of the yarn path in the convergence zone, and hence

$$
(\stackrel{\mathfrak{l}}{p}-\stackrel{\mathrm{q}}{q}) \cdot \nabla Q(\stackrel{\mathfrak{p}}{p})=0
$$

where of course the fell point is located on the mandrel surface,

$$
Q(\stackrel{\mathrm{I}}{p})=0 \text {. }
$$

These two equations are solved for each time step, subject to the constraints of zero fibre-fibre interaction before deposition and zero fibre slip after deposition. 


\section{SLIP CONDITIONS}

Consider a segment of a yarn in the $n s$ plane (fig.5), with $s$ as the coordinate in the length direction of the yarn and $n$ as the coordinate normal to the surface on which the yarn is deposited. The yarn tension is denoted as $F(s)$. Define $N^{\prime}$ as the normal load per unit length. Equilibrium considerations then lead to

$$
N^{\prime}=\frac{F}{R}=F \kappa_{n} .
$$

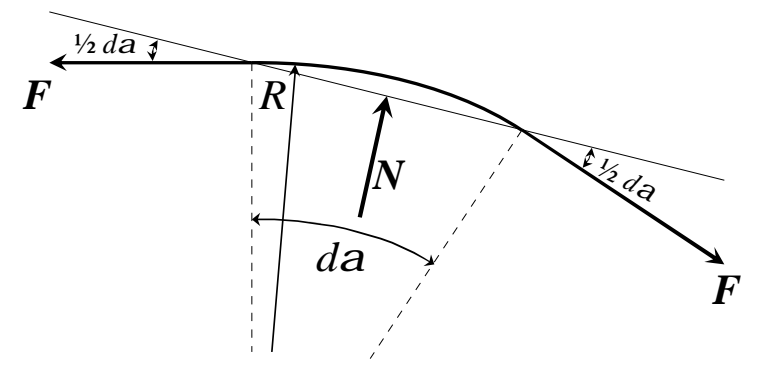

FIGURE 5. Tensile and normal forces acting on a curved yarn segment.

The same arguments can be followed in the $t s$ plane where the $t$ coordinate axis is normal to $s$ and $n$. In this case the friction force per unit length $S$ ' will balance the tension:

$$
S^{\prime}=F \kappa_{t} \text {. }
$$

Assuming Coulomb friction leads to

$$
S_{\text {max }}^{\prime}=\mu N^{\prime},
$$

with $\mu$ as the coefficient of friction, and hence

$$
\kappa_{t} \leq \mu \kappa_{n} .
$$

We will use this result for a slip indicator. The inplane curvature $\kappa_{t}$ is zero in case of zero friction, in which case the path is called geodesic, following the shortest path on the surface from one point to another.

\section{DISCRETE APPROACH}

We start from a triangular surface mesh, e.g. an STL representation or an FE mesh of the surface of the mandrel (fig.6). Each triangle is denoted as an element with nodes and edges. The sequence of node numbers connected to an element defines the normal vector of the element. The elements are numbered such that all element normals are pointing outwards. 

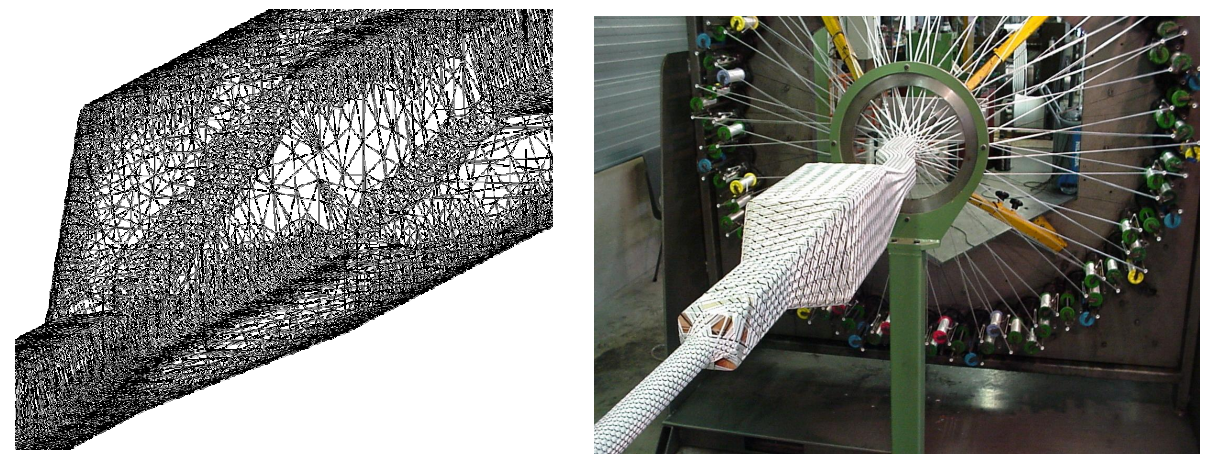

FIGURE 6. Validator mandrel; left: triangular surface mesh, right: in the braiding machine.

The fibre path is defined as an array of points. These points are always located exactly at an element edge. A time stepping procedure is used, where the relative positions of the spool plane, the spools and the supply points are updated in every step. The fibre path is progressed in a stepwise manner. Consider the geometry in fig.7. The current fell point $p^{(j)}$ is known, as well as the edge on which the fell point is located, and the connected 'free element' $\boldsymbol{a b c}$ where the fibre has not yet been deposited. The location of the supply point $\stackrel{q}{q}$ changes due to the relative motion of the spools during a time step $\Delta t$. The line from the current fell point $\underset{p}{\mathbf{r}^{(j)}}$ to the new supply point $\stackrel{\mathbb{r}}{q}^{(j+1)}$ can be above or below the free element (based on the element normal). In the latter case, a new fell point is determined using the geodesic path from the previous fell point to the new supply point via the appropriate free edge (ac or $\boldsymbol{b c}$ ) of the element.

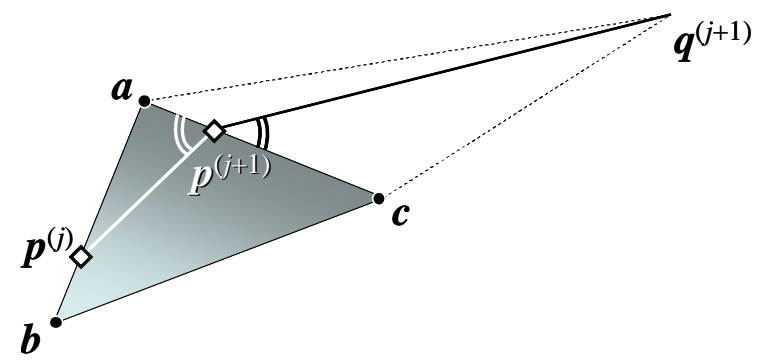

FIGURE 7. Location of the new fell point $\stackrel{\mathbf{I}}{(j+1)}^{(j+1)}$ between $\stackrel{\mathrm{I}}{p}^{(j)}$ and the supply point $\stackrel{\mathrm{I}}{q}^{(j+1)}$.

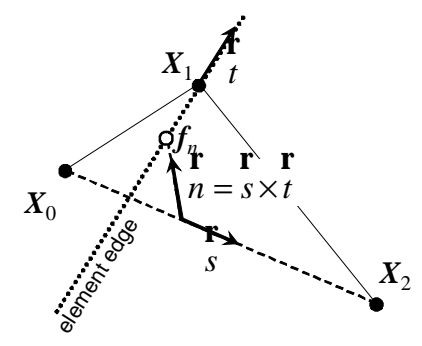

FIGURE 8. Discretised yarn trajectory on two planar elements.

Now consider a discrete yarn trajectory determined by the points $X_{0}, X_{1}$ and $X_{2}$, as depicted in fig.8. The unit vectors $t$ and $s$ respectively describe the directions of the edge between two elements and the line $l$ connecting $X_{0}$ and $X_{2}$. The vector $n$ is normal to $t$ and $s$. We will use the function $f(s)$ to represent the 3D yarn trajectory in the $(s, t, n)$ coordinate system as a function of a scalar $s$ increasing monotonically along $l$. The projection of $f(s)$ on $n$ determines the shortest path (or: geodesic) on the element surfaces from point $X_{0}$ to $X_{2}$.

This is the path the yarn will attain for zero friction conditions, which solves the deposition problem i.e. $p^{(j+1)}$ in fig.7. The new fell point is on the edge ac if this intersection is between the nodes $a$ and $c$. Otherwise the fell point will be on the edge $\boldsymbol{b} \boldsymbol{c}$ where it can be determined likewise. The procedure is repeated for the new fell point, the corresponding edge and the free element until no further intersections of the yarn with the mandrel surface are found. The time stepping procedure ends when all fell points have reached the other end of the mandrel. 


\section{SLIP PREDICTION}

Returning to the arbitrary yarn trajectory in fig.8, the normal and the inplane curvature are defined as, respectively,

with

$$
\kappa_{n}=\frac{\partial^{2} f_{n}(s)}{\partial s^{2}}, \quad \kappa_{t}=\frac{\partial^{2} f_{t}(s)}{\partial s^{2}}
$$

$$
f_{n}=\stackrel{u}{f} \cdot \dot{n}, \quad f_{t}=\stackrel{u}{f} \cdot \dot{t} .
$$

This is readily discretised to

$$
\kappa_{n} \cong \frac{2}{s_{2}-s_{0}} \cdot\left(\frac{f_{n}\left(s_{2}\right)-f_{n}\left(s_{1}\right)}{s_{2}-s_{1}}-\frac{f_{n}\left(s_{1}\right)-f_{n}\left(s_{0}\right)}{s_{1}-s_{0}}\right)
$$

and similarly for $\kappa_{t}$. Now interpret $X_{0,1,2}$ as $p^{(j-1),(j),(j+1)}$ respectively. Fibre slip is predicted if $\kappa_{t} \geq \mu \kappa_{n}$. This condition is checked after a new fell point has been determined.

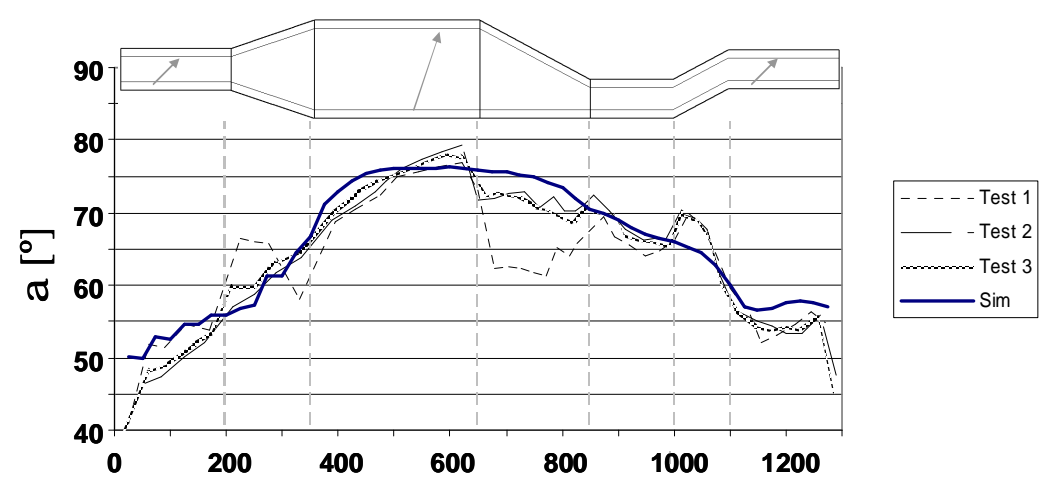

FIGURE 9. Braid angles (warp) vs axial coordinate: experimental and simulation results.

Earlier validations [5] showed significant effects of changing the friction conditions, see fig.9 (Test 1 without, Test $2 \& 3$ with adhesive tape). The agreement between experiment and simulation clearly improves when preventing slip. We will use these results to validate the slip indicator, which is currently being implemented.

\section{ACKNOWLEDGEMENT}

The support of EuroCarbon B.V. and the Dutch National Aerospace Laboratory NLR is gratefully acknowledged. Nico Tan provided valuable input to the slip indicator development.

\section{REFERENCES}

1. F.K. Ko. "Braiding" in Engineered Materials Handbook 1, ASM International, 1987, pp.519-528.

2. G.W. Du and P. Popper, Journal of the Textile Institute 85, 316-337 (1994).

3. Q. Zhang, D. Beale D. and R.M. Broughton, J of Manufacturing Sci. and Eng. 121, 345-350 (1999).

4. J.F.A. Kessels and R. Akkerman, Composites Part A 33, 1073-1081 (2002).

5. R. Akkerman and B.H. Villa Rodríguez, "Braiding Simulation for RTM Preforms", in Proceedings TEXCOMP-8, Nottingham (UK), CD edition, 2006. 